\title{
The Possible Inhibition Effect of Lycium barbarum Polysaccharides on Rat Intracerebral Hemorrhage Secondary Neuronal Apoptosis through Intervening Endogenous/Exogenous Pathways of Apoptosis
}

\author{
Yourui Zou' ShengYu Sun¹ Usama llyas Sharif² \\ ${ }^{1}$ Department of Neurosurgery, General Hospital of Ningxia Medical \\ University, Yinchuan, China \\ 2Department of Neurosurgery. General Hospital of Ningxia Medical \\ University, Yinchuan, China \\ ${ }^{3}$ Ningxia Key Laboratory for Cranio-cerebral Diseases, \\ Ningxia Medical University, Yinchuan, China \\ Indian J Neurosurg:2020;9:106-114
}

\begin{abstract}
Keywords

- Lycium barbarum

polysaccharides

- rat intracerebral hemorrhage

- neuronal apoptosis

- TNF- $\alpha$

$-\mathrm{BCl}-2$

Objective The aim of this study was to explore the inhibition effect and possible mechanism of Lycium barbarum polysaccharides (LBP) on rat intracerebral hemorrhage (ICH) secondary neuronal apoptosis.

Materials and Methods High-, medium-, and low-dose LBP (50, 100, and $200 \mathrm{mg} \bullet \mathrm{kg})$ and nimodipine $(10 \mathrm{mg} \bullet \mathrm{kg}$ ) groups were given once daily by 15 -day gavage before operation, while the sham operation and ICH groups were given the equal volume of saline. An ICH model was established by autologous blood injection and the neurological function in each group was scored at 4, 8, 12, 24, and 48 hours after modeling. Furthermore, terminal deoxynucleotidyl transferase dUTP nick end labeling analysis was performed to detect neuronal apoptosis, while western blot, immunohistochemistry, and real-time-polymerase chain reaction were used to study the influence of LBP on ICH secondary neuronal apoptosis.

Results The neurological function score was significantly decreased after $\mathrm{ICH}$, and the intervention effect of a single drug was not evident. The apoptotic nerve cells increased significantly in the ICH group but decreased considerably in the LBP groups. Furthermore, tumor necrosis factor alpha (TNF- $\alpha$ ) expression decreased significantly, while B-cell lymphoma 2 expression increased substantially in the high- and medium-dose LBP groups compared with ICH group, suggesting that LBP could reduce the effect of ICH. However, the impact of LBP did not correlate positively with the dose. Conclusion The application of LBP may not significantly improve neurological function after $\mathrm{ICH}$, but it can inhibit rat $\mathrm{ICH}$ secondary neuronal apoptosis.
\end{abstract}

Address for correspondence Hui Ma, MD, PhD, Department of Neurosurgery, General Hospital of Ningxia Medical University, Yinchuan, China (e-mail: Mahui0528@aliyun.com).

\section{Introduction}

Intracerebral hemorrhage (ICH) is one of the most devastating types of stroke, accounting for approximately 10 to $15 \%$ of all strokes. Patients with ICH have a poor prognosis. Eighty percent of the survivors beyond the acute phase may suffer from prolonged neurological deficit and brain atrophies. ${ }^{1}$ With the increasing aging population, the morbidity of ICH increases yearly, severely threatening middle-aged and elderly people's health.
The physio-pathologic mechanism of ICH is complicated, and secondary injury after ICH is an important factor that leads to a neurological deficit. Recent research shows that apoptosis is also involved in ICH secondary injury. ${ }^{2,3}$ Apoptosis, also known as programmed cell death, is an active cell suicide process that is essential for the maintenance of balance in organisms, after receiving some signals or stimulation. ${ }^{4}$ Studies on this and related topics have become the front edge 
and hotspot in bioscience. In this study, we investigated the endogenous and exogenous pathways of apoptosis.

Currently, drugs and surgery are used for the treatment of ICH in clinics. These two treatment methods complement each other and cause a decrease in the mortality and disability rate of ICH patients..$^{5-8}$ However, no matter which therapy is chosen, early prevention of ICH cannot be ignored. Thus, seeking an effective preventive neuroprotective drug is very urgent and crucial. Lycium barbarum is a traditional Chinese medicine that has Lycium barbarum polysaccharides (LBP) as an active ingredient. Modern pharmacological research has shown that LBP has many pharmacological effects, including free radical scavenging, oxidation resistance, antiaging, antitumor, neuroregulation, and immunoregulation. ${ }^{9,10}$ LBP can prevent neurovirulence caused by $\beta$-amyloid peptide by inhibiting c-Jun $\mathrm{N}$-terminal kinases (JNK), protein kinase RNA (PKR), and caspase-3 proapoptotic signaling pathways. ${ }^{11}$ It possesses protective effect against neurological deficit caused by ischemia and ischemia-reperfusion, the breakdown of blood-retinal barrier and oxidative stress injury, and remit neuronal apoptosis and retinal swelling. ${ }^{12}$ LBP can decrease the phosphorylation of JNK by decreasing lactate dehydrogenase secretion and inhibiting the activation of $\beta$-amyloid peptide enzyme to prevent apoptosis of nerve cells, decrease RNA-dependent PKR phosphorylation, and play a neuroprotective role. ${ }^{13}$ Recently, Wang et al found that LBP could decrease neuron apoptosis caused by cerebral ischemia by influencing mitochondrial apoptosis pathway. ${ }^{14}$ However, reports regarding the impact of LBP on ICH secondary neuronal apoptosis are limited. Therefore, in this study, we explored the preventive effect of LBP on the apoptosis of ICH secondary nerve cells and the possible mechanisms, which will form the theoretical basis for developing ICH-related preventive neuroprotective drugs.

\section{Materials and Methods}

\section{Animals and Drug Preparation}

LBP was supplied by Yinchuan Taifeng Biological Technology Co. Ltd. Rats were retrieved from the animal center of Ningxia Medical University and all the experiments were approved by the Institutional Animal Care and Use Committee of Ningxia Medical University. The healthy male SD rats ( $n=90$ ) weighing 180 to 230 g were randomly divided into sham, model, small-dose LBP, moderate-dose LBP, high-dose LBP, and nimodipine groups, with 15 rats in each group. Nimodipine has been widely used as a neuroprotective drug for ICH in clinical practice. ${ }^{15}$ Studies confirm that nimodipine provides neuroprotection by reducing cerebral edema ${ }^{16}$ and alleviating neuronal apoptosis. ${ }^{17}$ So, we chose nimodipine to design a positive drug group. Preoperatively, the smalldose LBP, moderate-dose LBP, and high-dose LBP groups were given 50,100 , and $200 \mathrm{mg} / \mathrm{kg}$ LBP by gavage. Rats in the nimodipine group were given $40 \mathrm{mg} / \mathrm{kg}$ nimodipine by gavage. The sham and model groups were given the same amount of saline instead, once daily.

\section{Intracerebral Hemorrhage Model}

We compared the various production methods of $\mathrm{ICH}$ and finally selected the second injection model. ${ }^{18}$ On this basis, we increased the modeling methods of Zhou et al. ${ }^{19}$ Each rat was anesthetized with chloral hydrate $(38 \mathrm{mg} / \mathrm{kg}$ intraperitoneally) and placed in a stereotactic frame (Narishige Instruments, Tokyo, Japan). A 30-gauge needle was introduced through a burr hole into the caudate nucleus of the left basal ganglia ( $3 \mathrm{~mm}$ lateral to midline, $0.6 \mathrm{~mm}$ posterior to bregma, $5.8 \mathrm{~mm}$ below the surface of the skull). The rats in the model, small-dose LBP, moderate-dose LBP, high-dose LBP, and nimodipine groups were slowly injected with $60 \mu \mathrm{L}$ of autologous blood without anticoagulant twice (interval: 2 min), while those in the sham group received a $60 \mu \mathrm{L}$ infusion of saline without collagenase or heparin before surgery. Five minutes after the injection, the needle was slowly withdrawn. After the surgery, the burr hole was sealed with bone wax. The wounds were closed with sutures after sterilization, and the animals were placed in warm boxes with free access to food and water.

\section{Rat Neurological Function Score}

The neurological function of the rats was scored according to Garcia et al's Neurological Deficit Scale. ${ }^{20}$ Before modeling, the preoperative neurological function of each rat was confirmed as good by primary scoring. After modeling, the scoring was repeated after the effect of the anesthesia wore off to exclude the rats that failed the modeling (score $>12 /$ death). The data loss was compensated by the spare rats. Later, the accurate neurological function scoring was performed at 4, 8, 12, 24, and 48 hours after modeling by a single blind method ( - Fig. 1).

\section{Tissue Perfusion, Fixation, and Paraffin Sections}

Chloral hydrate was intraperitoneally injected as an anesthesia (38 mg/kg) 48 hours after modeling, and 0.9\% saline $(\sim 150 \mathrm{~mL})$ and $4 \%$ paraformaldehyde $(\sim 300 \mathrm{~mL})$ were also injected. The rats were killed by decapitation, and their brains were taken out. Rhinencephalon, cerebellum, and low brain stem were removed, and the brain tissue in front or behind the needle was taken to prepare paraffin sections. The brain tissue sections were further prepared to perform

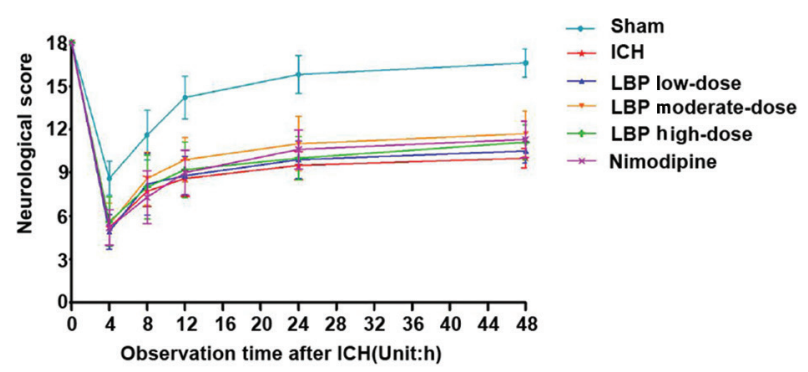

Fig. 1 Neurological function scores in each group. After modeling, neurological function score was performed at $4,8,12,24$, and 48 hours. The score of 18 at 0 hour indicated that the preoperative neurological function was good, and different broken lines represented different groups $(n=12)$. LBP, Lycium barbarum polysaccharides; ICH, intracerebral hemorrhage. 
the terminal deoxynucleotidyl transferase dUTP nick end labeling (TUNEL) and immunohistochemical experiments.

\section{Apoptosis Detection by TUNEL}

TUNEL is the most sensitive, rapid, and specific method used to detect apoptosis in situ. ${ }^{21}$ TUNEL kit (Roche) was used to detect apoptosis. After dewaxing and hydration, the paraffin sections were further incubated with proteinase $\mathrm{K}$ for 20 minutes at room temperature. Then, TUNEL working solution was added for another 60 minutes at $37^{\circ} \mathrm{C}$, and they were finally stained with 4,6- diamidino-2-phenylindole (DAPI). The results were observed and analyzed under a fluorescence microscope (all the cell nucleus showed blue fluorescence while apoptotic cells showed green fluorescence). In the ICH region, six views at high magnification were randomly photographed to analyze positive apoptosis rate and draw a histogram.

\section{Immunohistochemistry}

The tissue sections were placed in sodium citrate buffer to perform antigen retrieval. Then, $3 \% \mathrm{H}_{2} \mathrm{O}_{2}$ was added for 10 minutes at room temperature and $10 \% \mathrm{BSA}$ at $37^{\circ} \mathrm{C}$. Primary antibody was added overnight at $4^{\circ} \mathrm{C}$ (B-cell lymphoma 2 [Bcl-2], 1:100; tumor necrosis factor alpha [TNF- $\alpha$ ], 1:50; Abcam) and secondary antibody (goat antirabbit immunoglobulin G-horseradish peroxidase) for 20 minutes at $37^{\circ} \mathrm{C}$. DAB Color Developing Reagent Kit was used to detect the antigen-antibody reaction. The sections were stained with hematoxylin, blocked by neutral gum, and observed under a microscope.

\section{Western Blot Analysis}

At 48 hours after ICH, the rats were killed by decapitation and their brains were taken out. The hematocele was washed out with cool saline, and the tissues around ICH $(100 \mathrm{mg})$ were taken to extract whole protein. The protein concentration was detected by bicinchoninic acid, and the concentration was adjusted to make the loading amount consistent. The extracted whole protein was separated by sodium dodecyl sulfate-polyacrylamide gel electrophoresis (SDS-PAGE) and transferred to polyvinylidene difluoride (PVDF) membrane. After being blocked with $5 \%$ skimmed milk for 2 hours at room temperature, primary antibody was added (rabbit antirat Bcl-2, 1:500; mouse anti-rat TNF- $\alpha, 1: 200$, Abcam) overnight at $4^{\circ} \mathrm{C}$ and Glyceraldehyde 3-phosphate dehydrogenase (GAPDH) was used as internal reference. On the second day, the membrane was washed three times with tris-buffered saline (TBST) (5 minutes). Moreover, goat antirabbit/rabbit antimouse fluorescence secondary antibody (1:5000, Proteintech) was added at room temperature for 1 hour. Then, it was washed with TBST and scanned with Odyssey infrared imaging system. The gray value was processed and analyzed by Gelpro. ${ }^{22}$

\section{Real-Time Quantitative PCR}

At 48 hours after ICH, the rats were killed by decapitation; their brains were taken out and stored at $-80^{\circ} \mathrm{C}$. The total RNA was extracted with Trizol, and the OD was detected with an ultraviolet spectrophotometer at 260 and $280 \mathrm{~nm}$ to confirm the extracted RNA concentration and purification. The extracted RNA was transcribed to cDNA, and the expression levels of Bcl-2 and TNF- $\alpha$ were detected by real-time polymerase chain reaction (RT-PCR). The primer was designed, and the reaction system was $25 \mu \mathrm{L}$ : Maxima SYBR Green/ROX qPCR Master Mix (2×) $12.5 \mu \mathrm{L}$, DNA template, rat Bcl-2/TNF- $\alpha /$ GAPDH upstream and downstream primers, and water without nuclease. The system was placed in precooled tubes, and thermal cycle amplification was started after centrifugation: pretreated for 2 minutes at $50^{\circ} \mathrm{C}, 1$ cycle; predegeneration for 2 minutes at $95^{\circ} \mathrm{C}, 1$ cycle; degeneration for 10 minutes at $95^{\circ} \mathrm{C}$, annealing for 30 seconds at 60 annealing, extending for 30 seconds at $72^{\circ} \mathrm{C}, 39$ cycles. GAPDH was used as an internal reference and 2- $\Delta \Delta \mathrm{Ct}$ method ${ }^{22}$ was used to quantitatively analyze and draw box plots.

\section{Statistical Analysis}

The data were expressed as mean \pm standard error of mean. The data with the homogeneity of variance and normal distribution were analyzed by analysis of variance, while the data with the heterogeneity of variance or non-normal distribution were analyzed by Kruskal-Wills $\mathrm{H}$ test. The comparison was analyzed by SPSS 19.0. A $p$-value $<0.05$ was considered to be statistically significant.

\section{Results}

\section{Influence of LBP on Rat Neurological Function Score after ICH}

The score of neurological function 4 hours after operation in each group was significantly lower than that before the operation, suggesting that ICH modeling was successful. Compared with the sham operation group, the scores of neurological function in other groups were significantly decreased $(p<0.05)$, indicating that ICH caused deterioration of neurological function. Within the first 24 hours after ICH, the scores improved over time and stabilized at 9 hours afterward, suggesting that the injury caused by ICH was difficult to heal or even irreparable. Compared with the ICH group, the neurological function scores in the LBP (high, medium, and low doses) and nimodipine groups were slightly increased, but the difference was not statistically significant $(p>0.05)$. This could not prove that LBP and nimodipine improved the function after ICH.

\section{Apoptosis Detection by TUNEL}

The results showed that fewer amounts of TUNEL positive cells were seen in the sham operation group, and they were distributed around the needle track. The positive cells significantly increased in other groups; they were mostly distributed in the bleeding region and surrounding brain tissues. Most TUNEL positive cells were nerve cells, and the rest were glia cells and vascular endothelium cells. Compared with the sham operation group, the TUNEL positive cells in the ICH group were significantly increased $(p<0.01)$, suggesting that mechanical stimulus could cause nerve cells apoptosis that was more obvious 
in the ICH group. Compared with the ICH group, TUNEL positive cell rates in the LBP groups and the nimodipine group were significantly decreased. Among them, the medium-dose LBP group was the most significant $(p<0.01$ or $p<0.05)$ ( - Fig. 2 ).

\section{Influence of LBP on TNF- $\alpha$ Expression}

To study the influence of LBP on TNF- $\alpha$ expression in nerve cells after ICH, western blot (WB), immunohistochemistry, and RT-PCR were performed. The WB result showed that the TNF- $\alpha$ expression level in rat brain tissues from the sham operation group was very low, while that in the ICH group was significantly increased $(p<0.01)$, suggesting that the expression level was increased after ICH. Compared with the ICH group, the expression levels of TNF- $\alpha$ were significantly decreased in the LBP groups, especially in the medium-dose LBP group ( $p<0.01$ or $p<0.05)$, indicating that LBP could decrease TNF- $\alpha$ expression in brain tissues after ICH (-Fig. 3). The immunohistochemistry result showed that less TNF- $\alpha$ positive cells were observed around the needle track in the sham operation group, but lots were observed in the ICH group, mainly distributed in the bleeding region and surrounding brain tissues. This suggests that mechanical stimulus could induce TNF- $\alpha$ expression in nerve cells.
The induction effect of ICH was stronger than that of single surgery insertion (of the Sham operation). Compared with ICH, the TNF- $\alpha$ positive cells in the LBP groups were significantly decreased $(p<0.01)$, suggesting that LBP could inhibit TNF- $\alpha$ expression in nerve cells (-Fig. 4). Finally, RT-PCR was used to detect TNF- $\alpha$ mRNA expression levels in the bleeding region. Compared with the sham operation group, the TNF- $\alpha$ mRNA expression level in the ICH group was significantly increased $(p<0.01)$. Compared with the ICH group, the levels in the LBP groups were significantly decreased $(p<0.05$ or $p<0.01)$, especially in the high-dose LBP group ( - Fig. 5A)

\section{Influence of LBP on Bcl-2 Expression}

To study the influence of LBP on Bcl-2 expression in nerve cells after ICH, WB, immunohistochemistry, and RT-PCR were also performed. WB result showed that Bcl-2 was expressed in rat brain tissues from the sham operation group, and the expression remained at the lower moderate level. The level in the ICH group was significantly lower than that in the sham operation group $(p<0.05)$, suggesting that the expression was decreased after ICH. Compared with the ICH group, the expression level in the low-dose LBP group had no significant increase $(p>0.05)$, but that in the

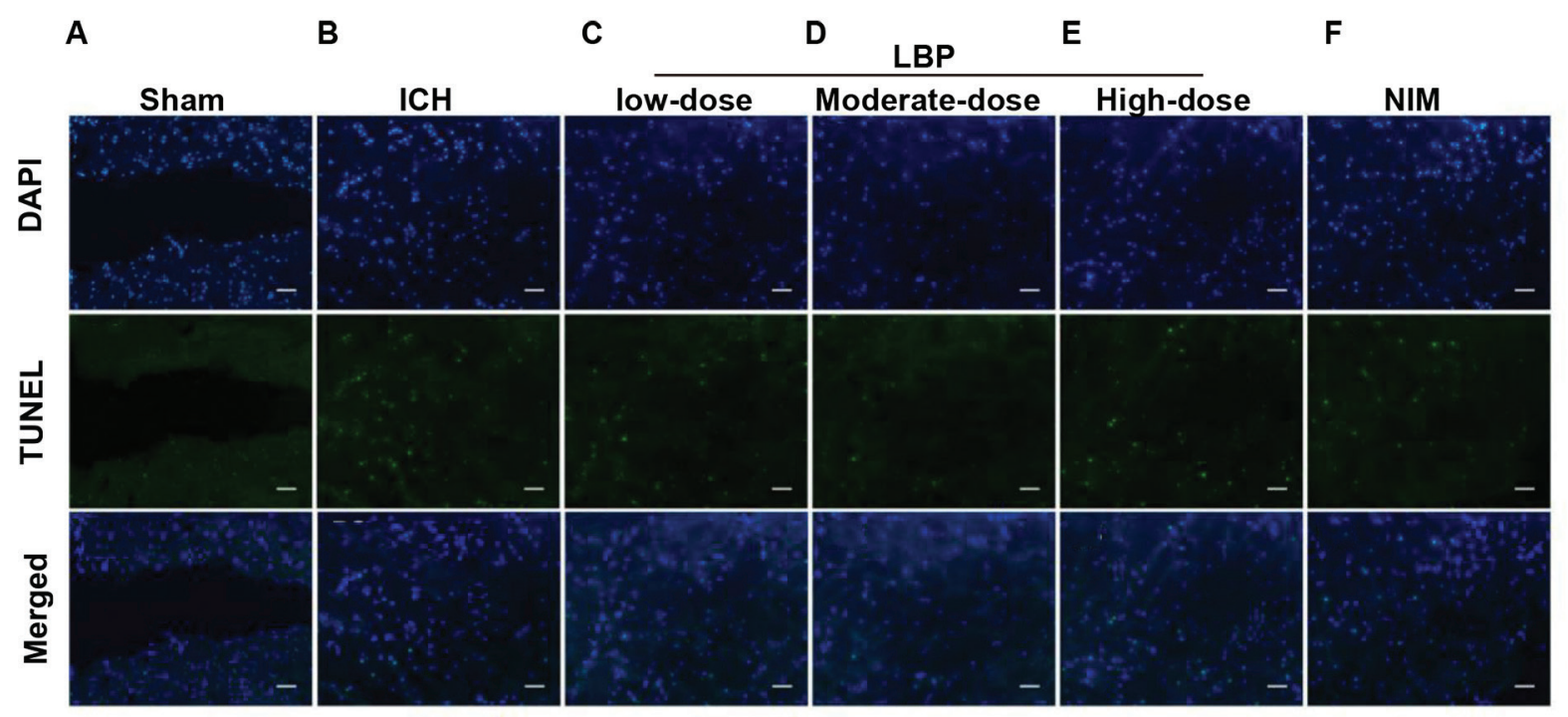

G

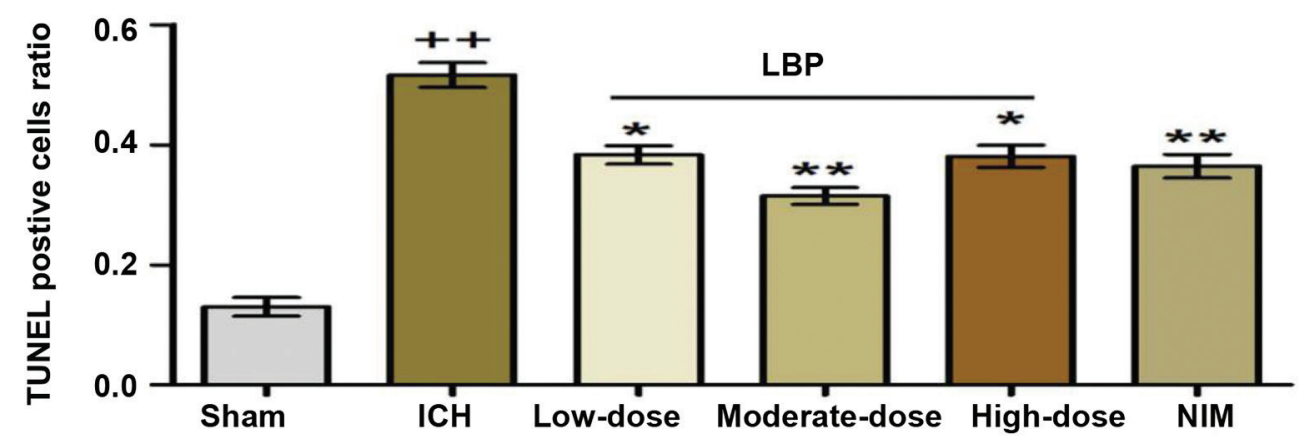

Fig. 2 TUNEL assay of apoptosis. Sham (A)/ICH (B)/low-dose LBP (C)/moderate-dose LBP (D)/hifh-dose LBP (E)/Nim (F), (A1-F1) TUNEL. (A2-F2) DAPI. (A3-F3) Merged images of TUNEL and DAPI. TUNEL-positive cells proportion of all cells in each visual field under a microscope (G). Results are expressed as mean \pm SEM $(n=6)$. $++p<0.01$, versus sham-operated group; ${ }^{*} p<0.05,{ }^{*} p<0.01$, versus ICH group. Scale bars, 25 um. LBP, Lycium barbarum polysaccharides; ICH, intracerebral hemorrhage; SEM standard error of mean. 
A

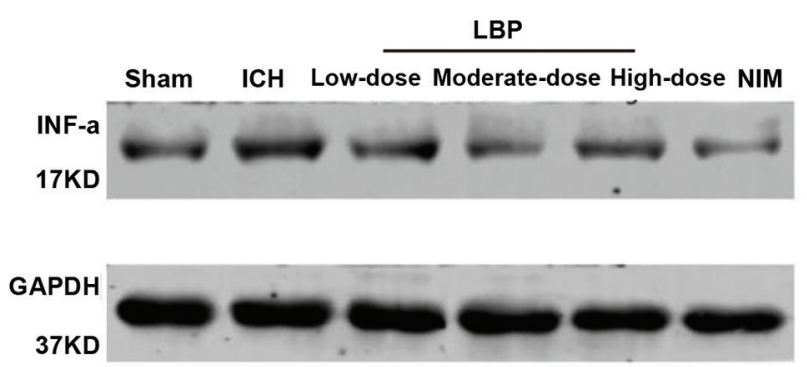

B

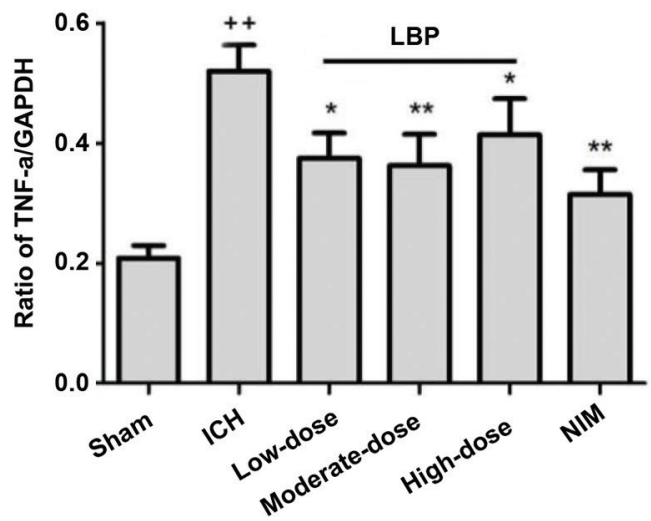

Fig. 3 Influence of LBP on TNF- $\alpha$ expression. (A) Western blot bands of TNF- $\alpha$ expression level; (B) TNF- $\alpha$ expression level in the bleeding region and surrounding brain tissues 48 hours after ICH. Results are expressed as mean \pm SEM $(n=6)$. $++p<0.01$, versus sham-operated group; \# $p>0.05,{ }^{*} p<0.05,{ }^{* *} p<0.01$, versus ICH group. ICH, intracerebral hemorrhage; LBP, Lycium barbarum polysaccharides; SEM standard error of mean; TNF- $\alpha$, tumor necrosis factor alpha.

A

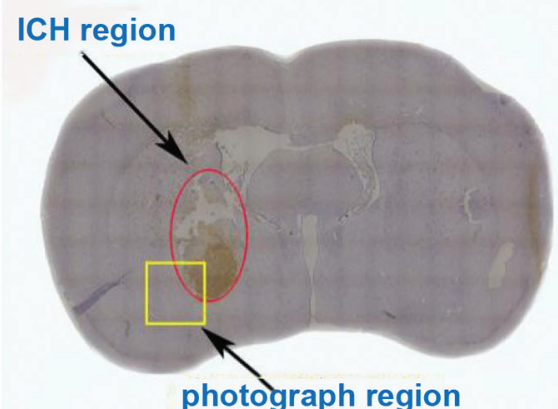

B
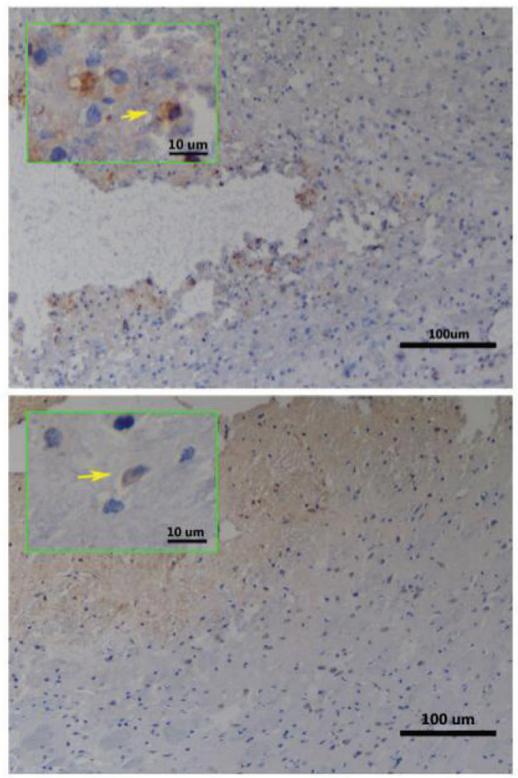

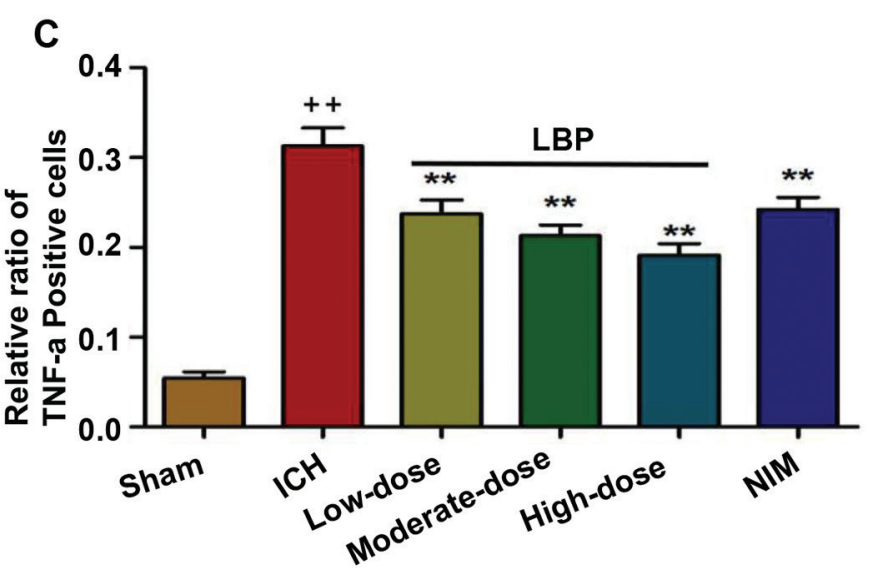

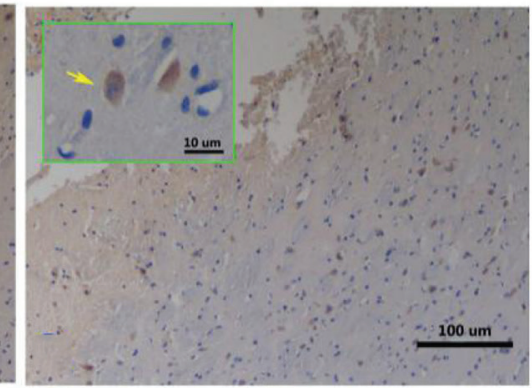
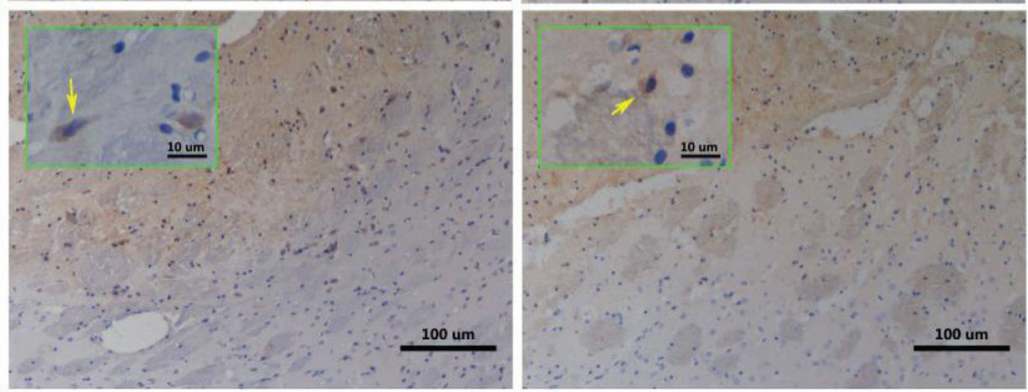

Fig. 4 TNF- $\alpha$ expression after ICH. (A): TNF- $\alpha$ expression. The ICH and photo regions were marked; (B1-B6) represented the expression of TNF- $\alpha$ from the sham operation, ICH, low-dose LBP, medium-dose LBP, high-dose LBP, and nimodipine groups. $\times 200$ (upper left), yellow arrow: TNF- $\alpha$ positive cells; $(\mathbf{C})$ : TNF- $\alpha$ positive cells rates in ICH regions from each group. The results are expressed as mean \pm SEM $(n=6)$. $++p<0.01$, versus sham-operated group; ${ }^{* *} p<0.01$, versus ICH group. ICH, intracerebral hemorrhage; LBP, Lycium barbarum polysaccharides; SEM standard error of mean; TNF- $\alpha$, tumor necrosis factor alpha. 
A

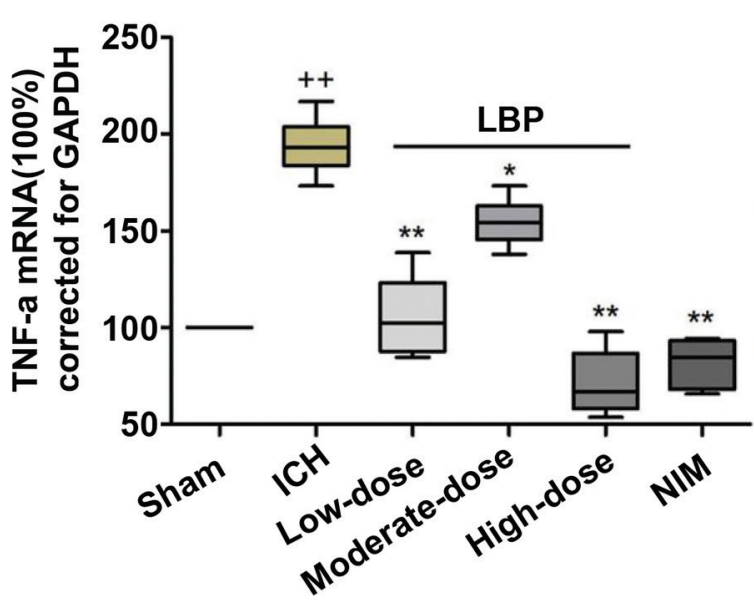

B

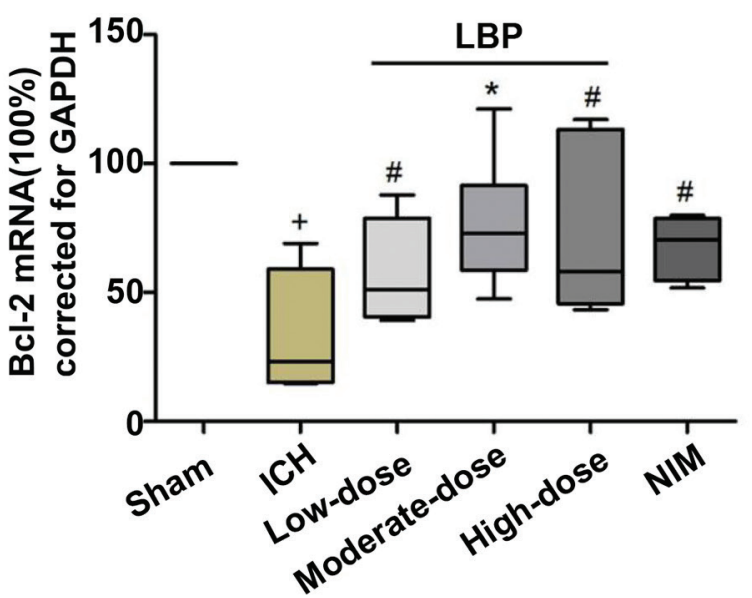

Fig. 5 TNF- $\alpha$ mRNA and Bcl-2 mRNA expression levels in brain tissues. (A) TNF- $\alpha$ mRNA expression in brain tissues 48 hours after ICH; (B) Bcl-2 mRNA expression in brain tissues 48 hours after ICH. The data presented were compared with mRNA levels in sham-operated animals that were put at $100 \%$. Data are presented as box plots with median/minimal/maximal whiskers. $+p<0.05,++p<0.01$, versus sham-operated group; $\# p>0.05,{ }^{*} p<0.05$, ${ }_{* *} p<0.01$ versus ICH group. BCl-2, B-cell lymphoma 2; ICH, intracerebral hemorrhage; LBP, Lycium barbarum polysaccharides; mRNA, messenger RNA; SEM standard error of mean; TNF- $\alpha$, tumor necrosis factor alpha.

A

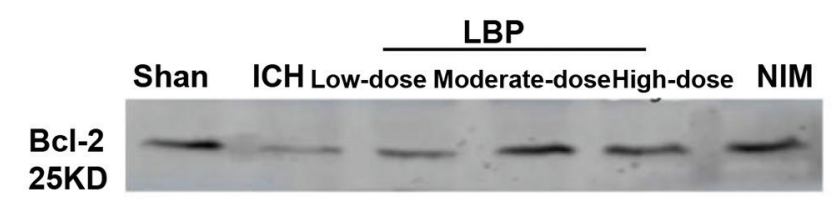

GAPDH 37KD
B

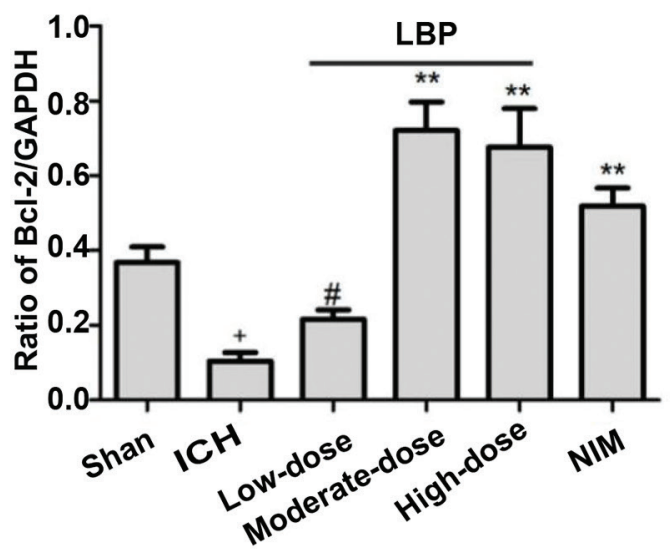

Fig. 6 Influence of LBP on Bcl-2 expression. (A) Western blot bands on Bcl-2 expression level. (B) The Bcl-2 expression level in brain tissues in the bleeding region 48 hours after ICH. The results are expressed as mean \pm SEM $(n=6) .+p<0.05$, versus sham-operated group; \# $p>0.05$, ** $p<0.01$, versus ICH group. Bcl-2, B-cell lymphoma 2; ICH, intracerebral hemorrhage; LBP, Lycium barbarum polysaccharides; SEM standard error of mean; TNF- $\alpha$, tumor necrosis factor alpha.

medium- and high-dose LBP groups increased significantly, suggesting that medium- and high-dose LBP could increase the expression after ICH ( - Fig. 6). The immunohistochemistry result indicated that Bcl-2 positive nerve cells were observed in the surroundings of the bleeding region from the ICH group, and the positive rate was approximately $20 \%$ (rate of positive cell number to total cell number under high amplification view), which was significantly decreased compared with the sham operation group $(p<0.05)$. It indicated that the $\mathrm{Bcl}-2$ expression in nerve cells was inhibited after ICH. Compared with the ICH group, the positive nerve cells (Bcl-2 expression) in the LBP groups were significantly increased $(p<0.01)$. The possible reason might be that $\mathrm{Bcl}-2$ expression in nerve cells was increased by LBP (absolute increase), and the apoptosis of $\mathrm{Bcl}-2$ expression in positive nerve cells was inhibited by LBP (relative increase) (-Fig. 7). Finally, RT-PCR was used to detect Bcl-2 mRNA expression levels in the bleeding region and surrounding brain tissues. Compared with the sham operation group, Bcl-2 mRNA expression level in the ICH group was significantly decreased $(p<0.05)$. Compared with the ICH group, the increases in the low- and high-dose LBP groups were not significant $(p>0.05)$, but that in the medium-dose LBP group was significant $(p<0.05)(-$ Fig. 5B). 
A

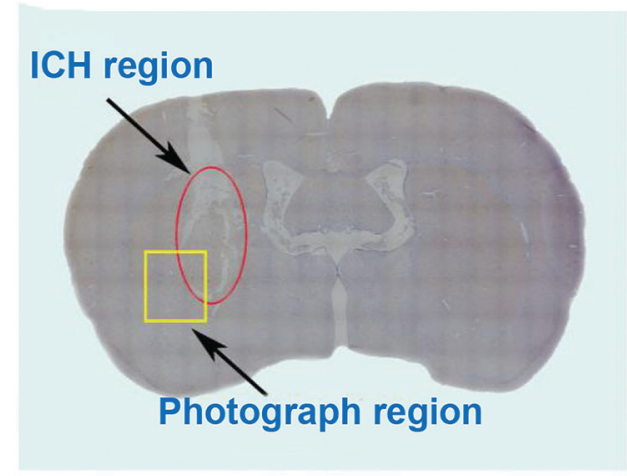

C

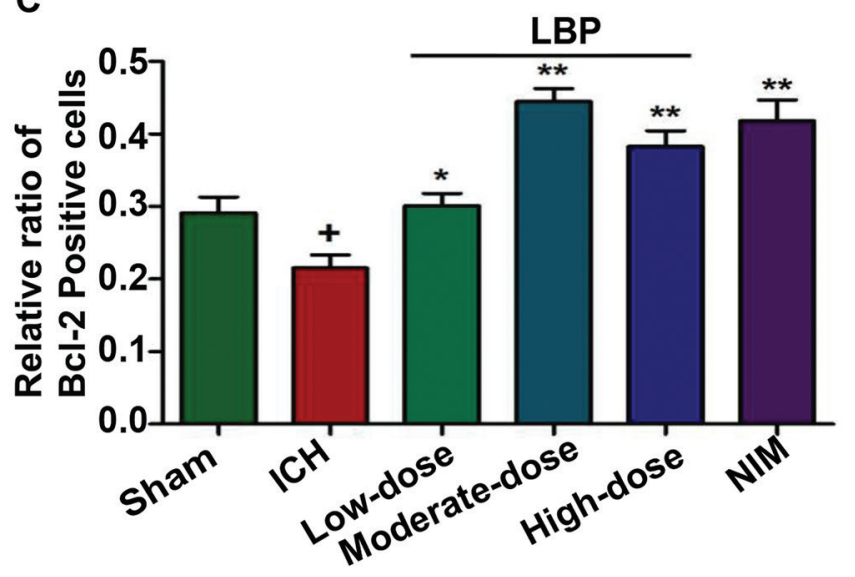

B
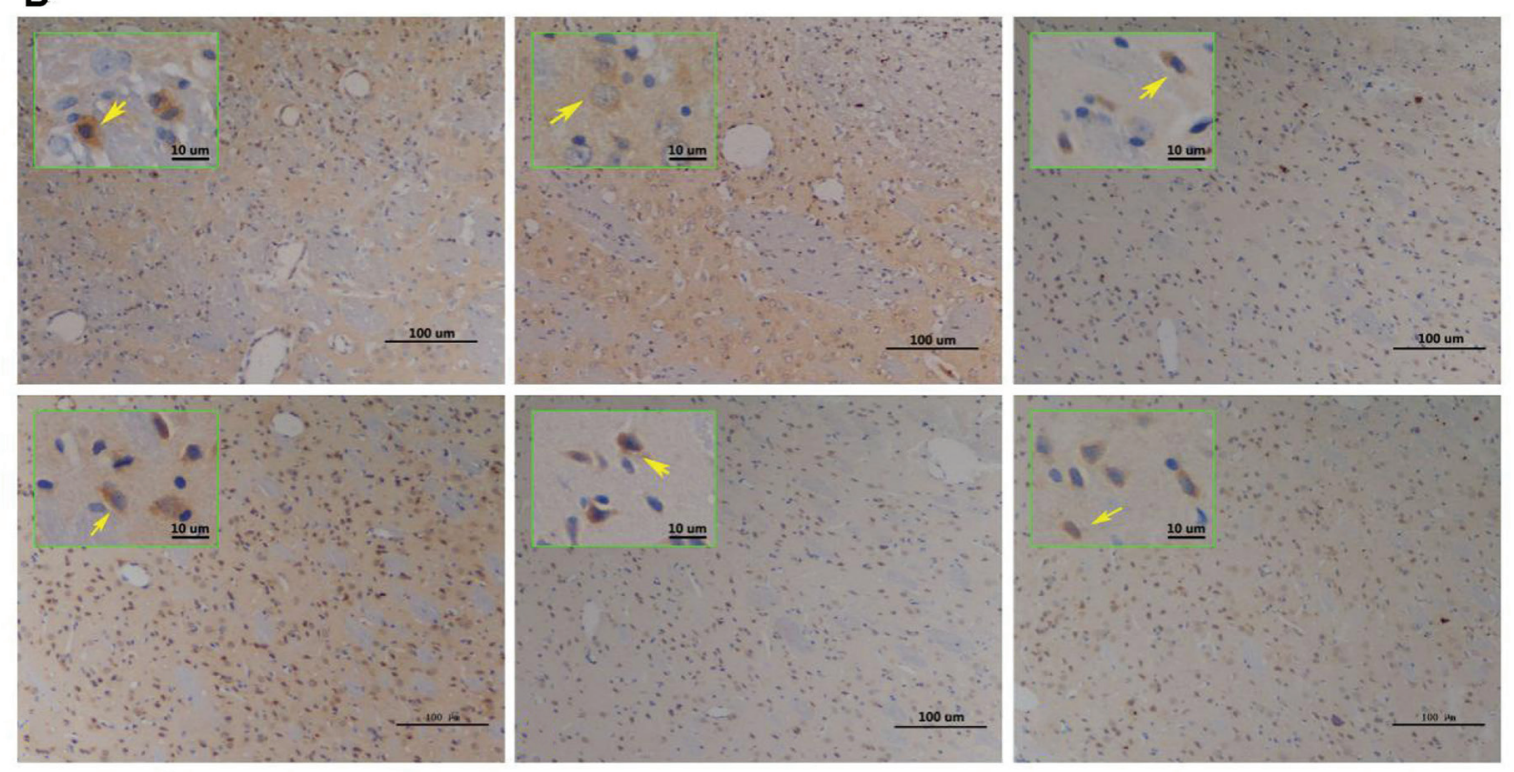

Fig. $7 \mathrm{Bcl}$-2 expression after ICH. (A) Bcl-2 expression. The ICH region and photo region were marked; (B1-B6) represent the expression of $\mathrm{BCl}-2$ from the sham operation, ICH, low-dose LBP, medium-dose LBP, high-dose LBP and nimodipine groups. $\times 200$ (upper left), yellow arrow: $\mathrm{Bcl}-2$ positive cells; (C) Bcl-2 positive cells rates in ICH regions from each group. The results are expressed as mean \pm SEM $(n=6) .+p<0.05$, versus sham-operated group; * $p<0.05,{ }^{* *} p<0.01$, versus ICH group. BCl-2, B-cell lymphoma 2; ICH, intracerebral hemorrhage; LBP, Lycium barbarum polysaccharides; SEM standard error of mean; TNF- $\alpha$, tumor necrosis factor alpha.

\section{Discussion}

ICH is a cerebrovascular disease with a high morbidity, and about one-third of the patients will show secondary neurological function deficit within a period after onset. ${ }^{23}$ The most intuitive performance is the changes in the neurological function score. By observing and analyzing the scores of neurological function at different time points after ICH, we found that ICH severely damaged neurological function, which could be significantly improved in the first 24 hours. However, the condition remained stable (plateau stage), meaning that ICH irreversibly injured the rat and the intervention of LBP could not remit such injury.

Apoptosis plays a very important role in the secondary injury of ICH. ${ }^{2,3}$ In our study, TUNEL analysis proved the existence of ICH secondary nerve cell apoptosis and the high occurrence rate in the ICH region and surrounding brain tissues that agree with other experiments. ${ }^{24-26}$ Also, our results confirmed that LBP could inhibit ICH secondary neuronal apoptosis, and this was more pronounced in the medium-dose LBP group. LBP protects brain tissues by inhibiting ICH secondary neuronal apoptosis. However, the apoptosis mechanism is very complicated, involving multipathways, of which exogenous and endogenous pathways are mostly studied. ${ }^{27,28}$

Exogenous pathway is mainly triggered by death receptor, and is also known as death receptor pathway. The death receptor contains death domain. For example, Fas-associated death domain can recruit and activate caspase-8, further leading to the activation of downstream effector and finally, apoptosis. ${ }^{29,30}$ TNF- $\alpha$ is the main factor mediating apoptotic exogenous pathway. ${ }^{31}$ Therefore, in our study, TNF- $\alpha$ was chosen to investigate the possible mechanism of ICH secondary neuronal apoptosis inhibition by LBP. 
In the endogenous pathway, the activation of caspase-9 (upstream factor) can cause cytochrome $\mathrm{C}$ release from mitochondrion, ${ }^{22,32}$ further inducing a series of apoptosis in the downstream. It is also called a mitochondrial pathway. The antiapoptotic members of the Bcl-2 family, including Bcl2 , can decrease apoptosis mainly by preventing mitochondrial outer membrane permeabilization, ${ }^{33}$ or by specifically blocking the morphologic features of apoptosis, including the plasma membrane blebbing, DNA cleavage, and nuclear condensation. ${ }^{34}$ The expression of $\mathrm{Bcl}-2$ is maintained at relatively high levels in sensory and sympathetic neurons in the adult peripheral nervous system. ${ }^{35,36}$ Thus, Bcl-2 was also chosen to explore the possible mechanisms of ICH secondary neuronal apoptosis inhibition by LBP in this study.

We examined the influences of LBP on Bcl-2 and TNF- $\alpha$ expression levels in brain tissues after ICH using three different techniques. The WB result showed that TNF- $\alpha$ expression level significantly decreased, while Bcl-2 expression level significantly increased in the LBP group, suggesting that LBP can greatly inhibit TNF- $\alpha$ expression ( - Fig. 3 ) and promote $\mathrm{Bcl}-2$ expression ( - Fig. 6). The immunohistochemistry result showed that TNF- $\alpha$ positive cell rate decreased, while that of $\mathrm{Bcl}-2$ increased in the LBP group, agreeing with the WB result. Also, the RT-PCR result showed that Bcl-2 mRNA expression level increased, while that of TNF- $\alpha$ mRNA decreased in the LBP group, further confirming that LBP promotes $\mathrm{Bcl}-2$ and inhibits TNF- $\alpha$ in the apoptotic pathway. However, the effect of LBP did not correlate positively with LBP dose. Thus, further investigation is required to determine the optimal LBP dose.

\section{Conclusion}

It has been proven that although the application of LBP did not significantly improve neurological function after ICH, it could still inhibit rat ICH secondary neuronal apoptosis. From both protein and gene levels, it was proved that LBP could influence TNF- $\alpha$ and Bcl- 2 expression after ICH. Furthermore, TNF- $\alpha$ and $\mathrm{Bcl}-2$ played important roles in exogenous and endogenous pathways of apoptosis. Thus, we conclude that LBP can inhibit ICH secondary neuronal apoptosis by interfering with the exogenous and endogenous pathways of apoptosis. The mechanism of apoptosis is very complicated, and cytokines involved interact with each other, forming a huge apoptotic pathway network. Therefore, the detailed mechanism by which LBP inhibits ICH secondary nerve cell apoptosis still needs further exploration.

\section{Funding}

This study was supported by Natural Science Foundation of China (Grant no. 81760449) and Natural Science Foundation of Ningxia (Grant no. NZ16127).

\section{Ethic Statement}

The study protocol was reviewed and approved by the Ethics Committees of the Institutional Animal Care and Use Committee of Ningxia Medical University.

\section{Conflict of Interest}

None declared.

\section{Acknowledgment}

None.

\section{References}

1 Naval NS, Nyquist PA, Carhuapoma JR. Management of spontaneous intracerebral hemorrhage. Neurol Clin 2008;26(2): 373-384, vii vii.

2 Hwang BY, Appelboom G, Ayer A, et al. Advances in neuroprotective strategies: potential therapies for intracerebral hemorrhage. Cerebrovasc Dis 2011;31(3):211-222

3 Qureshi AI, Suri MF, Ostrow PT, et al. Apoptosis as a form of cell death in intracerebral hemorrhage. Neurosurgery 2003;52(5):1041-1047, discussion 1047-1048

4 Krysko DV, Vanden Berghe T, D'Herde K, Vandenabeele P. Apoptosis and necrosis: detection, discrimination and phagocytosis. Methods 2008;44(3):205-221

5 Trabert J, Steiner T. Medical versus surgical management of intracerebral hematomas. Curr Atheroscler Rep 2012;14(4): 366-372

6 Tariq N, Adil MM, Saeed F, Chaudhry SA, Qureshi AI. Outcomes of thrombolytic treatment for acute ischemic stroke in dialysis-dependent patients in the United States. J Stroke Cerebrovasc Dis 2013;22(8):e354-e359

7 Siddiq F, Adil MM, Norby KE, Rahman HA, Qureshi AI. Rates and outcomes of neurosurgical treatment for postthrombolytic intracerebral hemorrhage in patients with acute ischemic stroke. World Neurosurg 2014;82(5):678-683

8 Li Q, Yang CH, Xu JG, Li H, You C. Surgical treatment for large spontaneous basal ganglia hemorrhage: retrospective analysis of 253 cases. Br J Neurosurg 2013;27(5):617-621

9 Chen Z, Kwong Huat Tan B, Chan SH. Activation of T lymphocytes by polysaccharide-protein complex from Lycium barbarum L. Int Immunopharmacol 2008;8(12):1663-1671

10 Miao Y, Xiao B, Jiang Z, et al. Growth inhibition and cell-cycle arrest of human gastric cancer cells by Lycium barbarum polysaccharide. Med Oncol 2010;27(3):785-790

11 Yu MS, Lai CS, Ho YS, et al. Characterization of the effects of anti-aging medicine Fructus lycii on beta-amyloid peptide neurotoxicity. Int J Mol Med 2007;20(2):261-268

12 Li SY, Yang D, Yeung CM, et al. Lycium barbarum polysaccharides reduce neuronal damage, blood-retinal barrier disruption and oxidative stress in retinal ischemia/reperfusion injury. PLoS One 2011;6(1):e16380

13 Ho YS, Yu MS, Yang XF, So KF, Yuen WH, Chang RC. Neuroprotective effects of polysaccharides from wolfberry, the fruits of Lycium barbarum, against homocysteine-induced toxicity in rat cortical neurons. J Alzheimers Dis 2010;19(3): 813-827

14 Wang T, Li Y, Wang Y, et al. Lycium barbarum polysaccharide prevents focal cerebral ischemic injury by inhibiting neuronal apoptosis in mice. PLoS One 2014;9(3):e90780

15 Raicević R, Jovicić A, Aleksić P, et al. [Role of nimodipine in the therapy of subarachnoid and intracerebral hemorrhage]. Vojnosanit Pregl 1999;56(6):593-598

16 Ma B, Zhang J. Nimodipine treatment to assess a modified mouse model of intracerebral hemorrhage. Brain Res 2006;1078(1):182-188

17 Korenkov AI, Pahnke J, Frei K, et al. Treatment with nimodipine or mannitol reduces programmed cell death and infarct size following focal cerebral ischemia. Neurosurg Rev 2000;23(3):145-150

18 Deinsberger W, Vogel J, Kuschinsky W, Auer LM, Böker DK. Experimental intracerebral hemorrhage: description of a double injection model in rats. Neurol Res 1996;18(5):475-477

19 Zhou ZH, Qu F, He X, et al. An improved autologous blood ICH model of rat: double injection and double withdrawal. Chin J Clin Neurosci 2004;4:408 
20 Garcia JH, Wagner S, Liu KF, Hu XJ. Neurological deficit and extent of neuronal necrosis attributable to middle cerebral artery occlusion in rats. Statistical validation. Stroke 1995;26(4):627-634, discussion 635

21 Li SY, Fu ZJ, Ma H, et al. Effect of lutein on retinal neurons and oxidative stress in a model of acute retinal ischemia/reperfusion. Invest Ophthalmol Vis Sci 2009;50(2):836-843

22 Li P, Nijhawan D, Budihardjo I, et al. Cytochrome c and dATP-dependent formation of Apaf-1/caspase-9 complex initiates an apoptotic protease cascade. Cell 1997;91(4):479-489

23 Hall NC, Packard BA, Hall CL. de Courten-Myers G, Wagner KR. Protein oxidation and enzyme susceptibility in white and gray matter with in vitro oxidative stress: relevance to brain injury from intracerebral hemorrhage. Cell Mol Biol 2000;46(3):673-683

24 Ardizzone TD, Zhan X, Ander BP, Sharp FR. SRC kinase inhibition improves acute outcomes after experimental intracerebral hemorrhage. Stroke 2007;38(5):1621-1625

25 Gong C, Boulis N, Qian J, Turner DE, Hoff JT, Keep RF. Intracerebral hemorrhage-induced neuronal death. Neurosurgery 2001;48(4):875-882, discussion 882-883

26 Qureshi AI, Ling GS, Khan J, et al. Quantitative analysis of injured, necrotic, and apoptotic cells in a new experimental model of intracerebral hemorrhage. Crit Care Med 2001;29(1):152-157

27 Solá S, Morgado AL, Rodrigues CM. Death receptors and mitochondria: two prime triggers of neural apoptosis and differentiation. Biochim Biophys Acta 2013;1830(1):2160-2166
28 Riedl SJ, Salvesen GS. The apoptosome: signalling platform of cell death. Nat Rev Mol Cell Biol 2007;8(5):405-413

29 Depraetere V, Golstein P. Fas and other cell death signaling pathways. Semin Immunol 1997;9(2):93-107

30 Kuang AA, Diehl GE, Zhang J, Winoto A. FADD is required for DR4- and DR5-mediated apoptosis: lack of trail-induced apoptosis in FADD-deficient mouse embryonic fibroblasts. J Biol Chem 2000;275(33):25065-25068

31 Locksley RM, Killeen N, Lenardo MJ. The TNF and TNF receptor superfamilies: integrating mammalian biology. Cell 2001;104(4):487-501

32 Hakem R, Hakem A, Duncan GS, et al. Differential requirement for caspase 9 in apoptotic pathways in vivo. Cell 1998;94(3):339-352

33 Youle RJ, Strasser A. The BCL-2 protein family: opposing activities that mediate cell death. Nat Rev Mol Cell Biol 2008;9(1):47-59

34 Ola MS, Nawaz M, Ahsan H. Role of Bcl-2 family proteins and caspases in the regulation of apoptosis. Mol Cell Biochem 2011;351(1-2):41-58

35 Merry DE, Veis DJ, Hickey WF, Korsmeyer SJ. bcl-2 protein expression is widespread in the developing nervous system and retained in the adult PNS. Development 1994;120(2):301-311

36 Merry DE, Korsmeyer SJ. Bcl-2 gene family in the nervous system. Annu Rev Neurosci 1997;20:245-267 